Matthias Stauch

\title{
Einschränkung des verwaltungsgerichtlichen Rechtsschutzes
}

\author{
I. Neuere Reformbemühungen zur VwGO
}

Keine sieben Monate nach Verkündung des 4. Gesetzes zur Änderung der VwGO (BGBl. I 1990, S. 2809) ist erneut ein Gesetzentwurf im Bundestag eingebracht worden, der einschneidende Umgestaltungen im System des verwaltungsgerichtlichen Rechtsschutzes anstrebt (Gesetzentwurf des Bundesrates vom 5.7.199I - BTDrucksache 12/1217 vom 27.9. 1991).

Bereits die 4. Novelle zur VwGO hat das gerichtliche Verfahren in wesentlichen Punkten neugeordnet. So ist das bisherige Kammerprinzip bei den Verwaltungsgerichten durch weitreichende Befugnisse des bestellten Berichterstatters im vorbereitenden Verfahren modifiziert worden ( $\$ \$ 87-87 \mathrm{~b}$ VwGO). Eine ganze Reihe von Entscheidungen trifft danach der Vorsitzende der Kammer oder der Berichterstatter praktisch als Einzelrichter.

In die VwGO sind weitreichende Präklusionsvorschriften aufgenommen worden ( $\$ 82$ Abs. 2 mit Ausschlußwirkung, $₫ 87$ b Abs. 3 mit Zurückweisungsbefugnis des Gerichts). Schließlich wurden die Instrumente des Entlastungsgesetzes (Gerichtsbescheid - $\$ 84-$, Zurückweisung der Berufung ohne mündliche Verhandlung und Absehen von der Darstellung der Entscheidungsgründe $-\mathbb{S}(\mathrm{z} 3 \mathrm{Oa}+\mathrm{b}$ ) dauerhaft in die Verfahrensordnung eingefügt. Eine Einführung der Zulassungsberufung ist bereits im Gesetzgebungsverfahren zur 4 . Novelle mit guten Gründen abgelehnt worden: Einem sehr geringen Entlastungseffekt steht dabei eine erhebliche Rechtsschutzeinbuße gegenüber.

\section{Der Gesetzentwurf vom 5.7. 199I}

Der jetzt eingebrachte Gesetzentwurf des Bundesrates sieht über die bisherigen Änderungen hinaus weitreichende Einschnitte im öffentlich-rechtlichen Rechtsschutz vor. Die Ländermehrheit im Bundesrat meint, damit könnten in erheblichem Umfang finanzielle Mittel freigesetzt werden, die die Länder zum Aufbau der Justiz in den neuen Ländern einsetzen wollen.

Der Gesetzentwurf ist also unter mehreren Gesichtspunkten kritisch zu betrachten. Erstens ist zu fragen, was die angestrebten Änderungen für den Rechtsschutz in der spezifischen Gerichtsbarkeit und unter ihren besonderen Funktionsbedingungen bedeuten, zweitens wird zu prüfen sein, ob die angenommene erhebliche Personaleinsparung mit der Novelle tatsächlich auch erreicht werden kann.

\section{Einzelrichterentscheidungen}

Der Gesetzentwurf des Bundesrates sieht eine Änderung von $₫ 6$ VwGO vor, nach der die Kammer den Rechtsstreit einem ihrer Mitglieder als Einzelrichter »überträgt «, wenn die Sache weder rechtlich noch tatsächlich schwierig und ohne grundsätzliche Bedeutung ist. Satz 2 des Abs. 4 der Regelung stellt klar, daß auf eine unterlassene Übertragung ein Rechtsbehelf nicht gestützt werden kann. Übertragungs- und Rückübertragungsbeschlüsse sind nicht anfechtbar.

Der letztlich im Bundesrat beschlossene Entwurf bleibt damit erheblich hinter ur- 
sprünglichen Ansätzen zur generellen Einführung des Einzelrichters in der Verwaltungsgerichtsbarkeit zurück. Der Rechtsstreit fällt weiterhin bei der Kammer an. Der Entwurf eröffnet den Kammern aber andererseits in allen öffentlich-rechtlichen Rechtsgebieten die Möglichkeit, den Einzelrichter einzusetzen. Das ist bisher lediglich in Asylstreitigkeiten $(\$ 31$ AsylVfG) der Fall gewesen. Der Wortlaut von $\$ 6$ Abs. I des Entwurfs und die Begründung (Drucksache 12/1217, S. 2 I und S. 5I) lassen erkennen, daß den Kammern bei der Entscheidung über die Übertragung auf den Einzelrichter kein Ermessen zustehen soll, obwohl auch betont wird, daß die Kammern in der Lage bleiben, den Besonderheiten des einzelnen Falls Rechnung zu tragen. Die Bundesregierung hat in ihrer Stellungnahme angemerkt, im weiteren Gesetzgebungsverfahren solle auch eine flexiblere »Kann-Regelung « erwogen werden.

Sollte der Entwurf des Bundesrates Gesetz und strikt in der historischen Auslegung nach den Gesetzesmotiven praktiziert werden, würde die Verwaltungsgerichtsbarkeit in ihrem Erscheinungsbild und der Art der bisherigen Rechtsschutzgewährung wesentlich umgestaltet werden.

Für das vorbereitende Verfahren ist bereits durch die 4. Novelle zur VwGO die Stellung des Berichterstatters oder des Vorsitzenden der Kammer der eines Einzelrichters für diesen Verfahrensabschnitt angenähert worden. Dies soll jetzt quasi auf den Bereich der mündlichen Verhandlung und der streitigen Entscheidung erweitert werden.

Für den Gesetzentwurf spricht, daß es in der Verwaltungsgerichtsbarkeit wie in anderen Gerichtszweigen einen nicht unerheblichen Anteil einfach gelagerter Routinefälle gibt, die im Grunde nicht der Befassung durch die Kammer bedürfen (z. B. Standardkonstellationen von Fahrerlaubnisentziehung, Fahrtenbuch, Abschleppkosten). Dafür ist eine flexible Übertragungsmöglichkeit auf den Einzelrichter wünschenswert und auch geeignet, die Kammer zu entlasten. Bei weiteren Fällen, die unter $\$ 6 \mathrm{Abs}$. I $\mathrm{VWG}_{\mathrm{w}} \mathrm{O}$ fallen würden wie Anerkennung als Kriegsdienstverweigerer, Ausweisung und Abschiebung von Ausländern und im Grunde auch die Entscheidung über das Asylrecht, stockt man m. E. zu Recht, von einer Entlastung der Kammer von Routinefällen zu sprechen.

$\mathrm{Zu}$ den Fällen der ersten Kategorie ist zu bemerken, daß bereits nach bisherigem Recht und auch bereits vor der 4 . Novelle eine erhebliche Zahl vor der mündlichen Verhandlung durch Hinweisschreiben oder Erörterungstermin vor einem Richter bzw. spätestens nach kurzer mündlicher Verhandlung durch Klagerücknahme beendet wurden. Der Spielraum für weitere »Rationalisierungs «-effekte ist daher sehr gering. Die Einzelrichterübertragung kann nur für die Fälle den Aufwand weiter verkürzen, in denen es nicht im vorbereitenden Verfahren zum Vergleich, zur Rücknahme oder einer anderen Erledigung kam. Auch für diesen Bereich ist ein möglicher Einspareffekt eher als gering zu veranschlagen. Kammern sind sehr effektiv und arbeitsteilig handelnde Organisationseinheiten. Es ist nicht so - wie Politiker und manchmal auch Ministerialbeamte vereinfachend glauben -, daß in einer Kammer jeder Fall praktisch dreimal vollständig von einem Berufsrichter bearbeitet würde und daß man mit der Übertragung auf den Einzelrichter die Arbeitskapazität praktisch zweier Berufsrichter freisetzen könnte. Vielmehr gibt es abgestimmte Rollen zwischen Berichterstatter, Vorsitzendem und zweitem Beisitzer, die eine richterliche Kontrolle des zu entscheidenden Sachverhalts in drei spezifischen Arbeitsformen beinhaltet. Nur der Berichterstatter und der Vorsitzende lesen die Akten. Dem zweiten Beisitzer wird der Sachverhalt in der Vorberatung mündlich vorgetragen. Dort trägt der Berichterstatter auch seine rechtliche Begutachtung vor. Der Vorsitzende erstellt zuvor in der Regel eine eigene Bewertung. Der zweite Beisitzer äußert sich 
nach dem Vortrag in der Vorberatung ohne spezielle eigene Vorbereitung auf den Fall. Die »Mehrarbeit« gegenüber dem Einzelrichtersystem besteht also darin, daß in jedem Fall die Akte zweimal gelesen wird. Die zweite rechtliche Beurteilung durch den Vorsitzenden ist im Normalfall bereits von geringerer Intensität als die des Berichterstatters. Für den zweiten Beisitzer fällt allein die Anwesenheit in der Vorberatung ins Gewicht. Durch diese Arbeitsweise wird bei geringer Mehrarbeit praktisch ein Optimum an Rechtsschutz erreicht, weil deutlich weniger Fehler, insbesondere bei der Sachverhaltsermittlung, aber auch bei der rechtlichen Einordnung, auftreten.

Diese eingehende Arbeitsweise ist aber nicht - wie oben dargelegt - in jedem Verfahren erforderlich. Die Wahl der geeigneten Arbeitsform sollte man dem Spruchkörper selbst überlassen, da der die Zweckmäßigkeit und Angemessenheit des Procedere aus eigener Kenntnis am besten einschätzen kann. Der Gesetzgeber sollte daher für alle öffentlich-rechtlichen Streitigkeiten nichtverfassungsrechtlicher Art $(\$ 40 \mathrm{VwGO})$ den fakultativen Einzelrichter durch eine "Kann «-Regelung in der VwGO zulassen. Dies zieht offenbar auch der BMJ in Erwägung. Die Verwaltungsgerichte könnten so noch vorhandene Möglichkeiten zur angemessenen Vereinfachung des Verfahrens maßvoll und zurückhaltend nutzen.

Gegen eine die Kammern bindende Einzelrichterzuweisung in einer Vielzahl von Fällen spricht auch - und das wird praktisch in der Diskussion des Gesetzentwurfs bisher kaum erwähnt -, daß die ehrenamtlichen Richter $(\$ 19$ VwGO) aus weiten Bereichen verwaltungsgerichtlicher Verfahren ausgeschieden würden. Das "Gesicht " des Verwaltungsprozesses würde sich dadurch entscheidend ändern. Die Kontrolle von Akten der öffentlichen Verwaltung würde eines wesentlichen demokratischen Elements beraubt werden. Bezogen auf den Gegenstand der Rechtsschutzgewährung sollte man diesen Verlust nicht als gering einschätzen. Die Spruchtätigkeit der Verwaltungsgerichte bezieht sich eng auf die Sphäre des Öffentlichen und des Politischen im Staat. Die eigenverantwortliche Mitwirkung von Bürgern und ihre Anwesenheit und Kontrolle auch im internen Entscheidungsprozeß der Gerichte schützt vor technokratischer Isolierung gerade bei den Angelegenheiten von öffentlichem Interesse und zwingt die Berufsrichter, ihre Erwägungen in Anwesenheit des Souveräns - des Volks - offenzulegen und zu begrïnden. Darauf sollte nur in Fällen von wirklich nachrangiger Bedeutung verzichtet werden.

\section{Fiktive Klagerücknahme}

Der Gesetzentwurf des Bundesrates sieht in $\$ 9^{2}$ Abs. 2 eine gesetzliche Fiktion der Klagerücknahme für den Fall vor, daß ein Kläger das Verfahren trotz Aufforderung drei Monate nicht betreibt. Dies betrifft die Verfahren, die bisher nach der Aktenordnung wegen Nichtbetreibens weggelegt worden sind. Dies hinderte bisher freilich einen Kläger nicht, das Verfahren später fortzuführen.

Der Entwurf knüpft an die Rücknahme an, weil es sich dabei um einen eindeutigen und feststehenden prozessualen Tatbestand handelt (Begründung zu Art. 8 Nr.9, S. 56 der Drucksache). Das mag zur Beendigung von Rechtsstreiten besonders effektiv erscheinen. $\mathrm{Ob}$ eine derart sachwidrige Verknüpfung eines Unterlassens im Prozeß mit einer klar typisierten Prozeßhandlung aber mit dem Rechtsstaatsprinzip noch vereinbar ist, erscheint mir zweifelhaft. Der Gesetzgeber ist bei der Wahl von gesetzlichen Fiktionen nicht völlig frei. Gesetzliche Fiktionen sind zulässig und in der Gesetzgebungstechnik gebräuchlich, um vergleichbare Sachverhalte einer Rechtsfolge zuzuführen, ohne sie unter gemeinsame Tatbestände fassen zu müssen. Völlig unvergleichbare, in wesentlichen Punkten verschiedene Sachverhalte dürfen 
aber nicht allein wegen der angestrebten Rechtsfolge durch Fiktion einer Regelung zugeführt werden. Es ist unverhältnismäßig und sachwidrig, an das Nichtbetreiben des Verfahrens ohne weitere Differenzierung die einschneidende Rechtsfolge der Klagrücknahme zu knüpfen. Eine wesentliche Entlastung wird nicht eintreten, weil in einer ganzen Reihe von Folgeverfahren mit Gewißheit über den Eintritt der Fiktion gestritten werden wird.

\section{Zulassungsberufung und gesetzliche Berufungsbegründungsfrist}

Der Gesetzentwurf des Bundesrates sieht in den $\mathbb{\$} \$ 124$ und 125 VwGO eine weitgehende Einschränkung der Berufung gegen verwaltungsgerichtliche Urteile vor. Die Berufung soll nur noch nach Zulassung durch das Verwaltungsgericht oder auf Nichtzulassungsbeschwerde durch das Oberverwaltungsgericht gegeben sein.

Die allgemeine Zulassungsberufung war bereits im Entwurf einer $\mathrm{VwPO}_{w} \mathrm{PS}$ I 4 I und 142 - BT-Drucksache 10/3437) enthalten und ist seitdem immer wieder in die Diskussion gebracht worden. Auf ihre Einführung ist zuletzt im 4 . VwGOÄndG nach erneuter Anhörung der Verbände verzichtet worden.

Das Institut der Zulassungsberufung ist sowohl durch die Verbände wie auch in der Literatur (siehe z.B. aus letzter Zeit Bethge, NJW 1991, 239I ff., 2398; Rennert, NJW I99I, I 2 ff., I s) eingehender Kritik unterzogen worden. Auch die Bundesregierung ist in ihrer Stellungnahme dem Entwurf des Bundesrates insoweit grundsätzlich entgegengetreten (BT-Drucksache I 2/ 1 $217^{-S}$. 72 f.). Dieser Kritik ist zuzustimmen.

Die Abschaffung einer zweiten Tatsacheninstanz würde zu einer wesentlichen Verkürzung des Rechtsschutzes führen, ohne daß diesen »Kosten « für die Rechtssuchenden ein wesentlicher »Nutzen « durch merkliche Personaleinsparungen entsprechen würde.

Es ist systemwidrig, den Zugang zum Berufungsverfahren entscheidend an Gründe zu knüpfen, die für das Revisionsverfahren gelten (grundsätzliche Bedeutung, Divergenz, Verfahrensfehler). Der die Gründe I, 2 und 4 erweiternde 3. Zulassungsgrund »besondere tatsächliche oder rechtliche Schwierigkeiten « der Rechtssache ist darüber hinaus so offen und untechnisch gefaßt, daß sich die Zulassung der Berufung einem relativ ungebundenen Annahmeverfahren durch die Oberverwaltungsgerichte annähern würde. Der eigentliche Rechtsstreit würde in das Beschwerdeverfahren über die Nichtzulassung der Berufung verlagert werden und dort unter abstrahierten formellen Gesichtspunkten mit vergleichbarem Aufwand ausgetragen werden. Da die Oberverwaltungsgerichte bereits weitgehende Möglichkeiten haben, bei wenig aussichtsreichen Berufungen mit wenig Aufwand zu entscheiden ( $\$ \$$ i joa und b VwGO - Zurückweisung der Berufung durch einstimmigen Beschluß ohne mündliche Verhandlung/Verzicht auf weitere Begründung), könnte es allenfalls zu sehr geringen Entlastungseffekten kommen. Dazu steht der Einschnitt in das bestehende Rechtsschutzsystem außer Verhältnis.

\section{Anwaltszwang vor den Oberverwaltungsgerichten}

Den Verfassern des Bundesratsentwurfs sind anscheinend selbst Restzweifel an der erhofften Wirkung der Zulassungsberufung geblieben. In der Begründung (Art. $8 \mathrm{zu}$ Nr. 10, S. 56) findet sich daher die Wendung, Zweifel am Entlastungseffekt könnten durch die Ausgestaltung des Verfahrens ausgeräumt werden. Der Entwurf sieht deshalb u. a. vor, vor den Oberverwaltungsgerichten den Anwaltszwang einzuführen $(\$ 67$ Abs. I). Dies zielt direkt auf eine materielle Erschwerung des Zugangs zum 
Rechtsschutz, die im wesentlichen sozial schwächere Bürger treffen wird. Die sach-

lichen Anforderungen des Verfahrens für sich erfordern nicht unbedingt eine anwaltliche Vertretung. Im Wege der Amtsermittlung klären die Oberverwaltungsgerichte den entscheidungserheblichen Sachverhalt auf, ein unvollständiger Sachvortrag eines Beteiligten führt für den Rechtssuchenden also nicht zu Nachteilen. Er erschwert aber auch für das Gericht das Verfahren nicht wesentlich, da das Gericht ohnehin von sich aus ermittelt und sich das Wesentliche in der Regel aus den Behördenakten ergibt. Im übrigen werden mutwillige Kläger durch den Anwaltszwang auch nicht vom Oberverwaltungsgericht ferngehalten. Wem das gerichtliche Verfahren auch nur einigermaßen vertraut ist, der weiß, daß er auch für die zweite Instanz einen Antrag auf Prozeßkostenhilfe und Beiordnung eines Rechtsanwaltes stellen kann. In diesem Fall muß das Oberverwaltungsgericht eingehend die Erfolgsaussichten des Rechtsmittels prüfen. Der Umfang der inhaltlichen Prüfung erreicht in der Praxis oft den einer Berufung, darüber hinaus sind auch die Einkommens- und Vermögensverhältnisse des Antragstellers in allen Einzelheiten (bis hin zu Unterhaltsansprüchen) zu untersuchen. Einsparungen von Personal oder eine merkliche Arbeitserleichterung wird der Anwaltszwang vor den Oberverwaltungsgerichten kaum bringen.

\section{Weitere Verfabrensverkürzung in Asylsachen}

Der Gesetzentwurf enthält im weiteren einige Änderungen zum Asylverfahrensrecht (u. a. Abschaffung der Beschwerde gegen Beschlüsse des Verwaltungsgerichts über Prozeßkostenhilfeanträge). Diese sind jedoch wegen des wesentlich weiterreichenden Gesetzentwurfs der Bundesregierung wohl als überholt anzusehen.

\section{Ausblick}

Der Entwurf des Bundesrates enthält keinerlei Prognose, wieviele Richter z. B. aus der Verwaltungsgerichtsbarkeit durch die vorgeschlagenen Regelungen freigesetzt werden können. Er läßt auch nicht erkennen, ob dazu bezogen auf die einzelnen Gerichtsbarkeiten tatsächlich ein konkreter Personalbedarf in den neuen Bundesländern besteht. Für die Arbeitsgerichtsbarkeit ist das allgemein bekannt, für andere Bereiche bedürfte dies zunächst der Prüfung, bevor so weitreichende Einschnitte im Rechtsschutzsystem vorgenommen werden wie jetzt vorgeschlagen.

Für die Verwaltungsgerichtsbarkeit ist festzustellen, daß die Umsetzung des Entwurfs neben aller vorgetragenen Einzelkritik auch die Tendenz zu einer weiteren Komplizierung des Rechtsschutzsystems vorantreibt. Durchgängige einfache Grundstrukturen mit klar gegliederten Funktionen für die Instanzen werden zugunsten von immer weiteren Spezialregelungen für einzelne Rechtsgebiete (bisher schon für das Asylrecht, Wehrrecht, Wohngeld- und Lastenausgleichsrecht) und einem ganzen Strauß besonderer förmlicher Anforderungen zurückgedrängt. Aus dem Rechtsschutz vor öffentlicher Gewalt könnte letztlich statt Wohltat Plage werden. Stelkens hat $\mathrm{m}$. E. zu Recht festgestellt, daß »die Gefahr besteht, daß eine verkürzte, auf das Finanzielle beschränkte Sicht den Blick auf den rechtsstaatlichen Wert einer Verwaltungsgerichtsbarkeit verwehrt...« (NVwZ 1991, 209ff., 219) und daß die Schere zwischen eingeräumten subjektiv-öffentlichen Rechten und den Möglichkeiten, sie tatsächlich durchzusetzen, sich immer weiter zu öffnen droht. 